73

Sonderdruck aus

Mathematische Nachrichten

Band 53 (1972)

Heît 1-6

AKADEMIE-VERLAG - BERLIN 


\title{
On right residuals in lattice ordered groupoids
}

\author{
By Farmenc A. SzÁsz of Budapest \\ To Professor LászLó KaLMár on his $65^{\text {th }}$ birthday
}

(Eingegangen am 18. 3. 1971)

Following G. BrRкноғт [2] and L. Fuchs [3, p. 191] a lattice ordered groupoid, or shortly a l. o. groupoid, is defined as a groupoid $G$, which is at the same time also a lattice, satisfying the distributivity requirements:

$$
(a \smile b) c=a c \cup b c \text { and } a(b \smile c)=a b \smile a c
$$

for every $a, b, c \in G$. The monotonity laws, i. e. $a \leqq b$ always implies $a c \leqq b c$ and $c a \leqq c b$, follow obviously from (1). Here the duals of (1) are neither assumed, nor can be derived from (1), therefore for 1. o. groupoids the duality principle fails generally to be valid. If we assume that for all $a, b \in G$ an element $c=a: b$ there exists such that

$$
x \leqq c \text { is equivalent to } b x \leqq a,
$$

then $c=a: b$ is called the right residual of $a$ and $b$, furthermore $G$ is said to be a right residuated 1 . o. groupoid. More generally, $a: b$ can be defined also in some partially ordered groupoids. The right residual generalizes the concept of the right-sided ideal quotient in the ring theory. Some properties of 1. o. groupoids and of right residuals are collected in G. BIRкноғF [2] and L. Fuchs [3].

If the l.o. groupoid $G$ is a complete lattice and it fulfils the infinite distributive laws:

$$
a\left(\bigvee_{\alpha} b_{\alpha}\right)=\bigvee_{\alpha} a b_{\alpha} \text { and }\left(\bigvee_{\alpha} b_{\alpha}\right) a=\bigvee_{\alpha} b_{\alpha} a,
$$

then $G$ is called a complete l. o. groupoid.

For results on some l. o. groupoids we refer the reader e. g. to B. A. Axmrunakinvich-Ju. M. Ruabukhin [1], G. Birkhoff [2], L. Fuchs [3], I. Lesieur [7], O. Steinfeld [8, 9, 10], I. V. Stelleckiy [11], E. G. Šul'GeYFer [12], F. Szísz [13], M. WARd [17] and M. WARd-R. P.Dilworth [18]. For notions of lattice theory see yet G. Szász [16]; furthermore for notions of ring theory and group theory see N. JAconson [4] and A. G. Kurosm [5], respectively. 
Obviously, an infinite union of right residuals of a complete residuated 1. o. groupoid $G$ fails generally to be again a right residual of some elements $a$ and $b$ of $G$.

On the other hand, if, in particular, $G$ is the 1. o. semigroup of all ideals (with respect to the usual lattice and semigroup operations) of an associative commutative principal ideal ring $A$ with unity element and without divisors of zero, then any finite union of arbitrary right residuals $a_{i}: b_{i}\left(a_{i}, b_{i} \in G\right.$, $i=1,2, \ldots, n)$ equals to a right residual $a: b$ for some $a$ and $b$ of $G$.

The purpose of this paper is to give a sufficient condition, which implies that any finite union of right residuals of $G$ is again a right residual. Our result has some applications to the theory of rings, of semigroups, of groups and of (rational or algebraic) numbers. axioms.

In what follows we assume that our 1. o. groupoid $G$ satisfies yet further

Axiom 1. $G$ contains a maximal element $e$ and a minimal element 0 , satisfying $x e=e x=x$ and $x 0=0 x=0$ for every $x \in G$.

Remark 2. Axiom 1 seems to be essentially different that the lattice $C_{r}$ is complete.

Proposition 3. The l. o. groupoid $G$, satisfying Axiom 1, is negatively ordered, i. e. $x y \leqq x \cap y$ holds for every $x, y \in G$.

Proof. We obtain by $x \leqq e, y \leqq e$ and by the monotonity laws

$$
x y \leqq e y=y \quad \text { and } \quad x y \leqq x e=x \text {, }
$$

consequently $x y \leqq x \frown y$.

Axiom 4. $G$ is a right residuated l. o. groupoid, $x: y$ denoting the right residual of $x$ and $y$.

Proposition 5. For $x \leqq y$ in a l.o. groupoid satisfying Axiom 1 holds $y: x=e$

Proof is by Axioms 1 and 4 trivial.

Axiom 6. For any nonzero element $x$ and nonzero element $y$, and arbitrary element $z(x, y, z \in G)$ of the right residuated 1. o. groupoid holds

$$
(x: y) \approx: x=z: y \text {. }
$$

Proposition 7. For any nonzero element $x$, and for arbitrary element $y$ of the l.o. groupoid, satisfying Axioms 1, 4 and 6, holds $x y: x=y$.

Proof. If $x: y=u$ and $y=e$, then we have $y u \leqq x$, that is

$$
u=e u=y u \leqq x,
$$

whence by $y x \leqq x$,

$$
x: y=u
$$

Now axiom 6 implis

$v=e v \leqq \approx$ to $\approx, \cos$

Proposition 8. $A n$. the left cancelling rule;

Proof. $x y=x \approx$,

$$
x y: x=?
$$

Proposition 9. $A$ r has no nonzero divison and $y \neq 0$ aluays im

Proof is, by Pro]

Axiom 10. For eve poid $G$ holds :

$$
((x \sim y): x
$$

Proposition 11. $F$ and 10 condition $x y$

Proof. If $x y=y$ $(x \cap y): x$

whence by Axiom 10

Proposition 12. Fo: $x \cup y=e$ and $x \cup z=$

$$
x \cup y \approx=a
$$

Proof is, by the re trivial.

Axiom 13. The la1 the 1. o. groupoid $G$, is and $L_{2}$, contained in

Remark 14. Obvic for arbitrary nonzero there exist such that of $G$ being contained

We can now form

Theorem 15. Let G and 13 , containing sor (4)

$$
x_{1} \cup y_{1}=e
$$


f a complete residuated dual of some elements $a$

semigroup of all ideals ations) of an associative nt and without divisors iduals $a_{i}: b_{i}\left(a_{i}, b_{i} \in G\right.$, some $a$ and $b$ of $G$.

ondition, which implies $\mathrm{n}$ a right residual. Our f semigroups, of groups

d $G$ satisfies yet further

a minimal element 0 , ry $x \in G$.

rent that the lattice $G$

Axiom 1, is negatively

monotonity laws

$\therefore y$ denoting the right

isfying Axiom 1 holds

s element $y$, and arbit. o. groupoid holds

or arbitrary element $y$ olds $x y: x=y$.

$u \leqq x$, that is whence by $y x \leqq x \frown y$ it follows also $x \leqq u \leqq x$, consequently

$$
x: y=u=x .
$$

Now axiom 6 implies $z: e=x z: x$, but $v=z: e$ equals by $z \leqq v$ and $v=e v \leqq z$ to $z$, consequently one has $x z: x=z$, indeed.

Proposition S. Any 7.o. groupoid satisfying the axioms 1, 4 and 6 fulfits the left cancelling rule, that is $x y=x z$ and $x=0$ imply $y=z$.

Proof. $x y=x \approx, x \neq 0$ and Proposition 7 imply $y=z$, being

$$
x y: x=y \text {. }
$$

Proposition 9. Any 7. o. groupoid $G$ satisfying the Axioms 1, 4 and 6 has no nonzero divisors of zero, furthernore $G$ is an Ore lattice, that is $x \neq 0$ and $y \neq 0$ aluays imply $x \cap y \neq 0$.

Proof is, by Propositions 8 and 3 , trivial.

Axiom 10. For every element $x$ and $y$ of the right residuated 1 . o. groupoid $G$ holds :

$$
((x \cap y): x) \cup((x \cap y): y)=e .
$$

Proposition 11. For a 7.o. groupoid $G$ satisfying the Axioms 1, 4, 6 and 10 condition $x y=y x=x \cap y$ implies $x \cup y=\epsilon$.

Proof. If $x y=y x=x \frown y$, then Proposition 7 vields

$$
(x \cap y): x=y \quad \text { and } \quad(x \cap y): y=x,
$$

whence by Axiom 10 we have $x \cup y=\epsilon$, indeed.

Proposition 12. For the 7 . o. groupoid $G$ satisfying Axiom 1 the conditions $x \cup y=e$ and $x \cup \approx=e$ imply

$$
x \cup y \approx=x \cup \approx y=\ell .
$$

Proof is, by the requirements (1), by $e^{2}=e$ and by the negative ordering, trivial.

Axiom 13. The lattice $\boldsymbol{L}$ of all left ideals, contained in $G^{2}=G$. $G$, of the 1. o. groupoid $G$, is an Ore lattice, that is for the nonzero left ideals $L_{1}$ and $L_{2}$, contained in $G^{2}$, of $G$ always $L_{1} \frown L_{2} \neq 0$ holds.

Remark 14. Obviously, Axiom 13 is equivalent to the condition that for arbitrary nonzero elements $g_{1}$ and $g_{2}$ of $G$ some elements $f_{1}$ and $f_{2}$ of $G$ there exist such that $f_{1} g_{1}=f_{2} g_{2} \neq 0$ holds, the left ideals $G g_{1}$ and $G g_{2}$ of $G$ being contained in $G^{2}=G . G$.

We can now formulate our

Theorem 15. Let $G$ be a 7 . o. groupoid satisfying the Axioms 1, 4, 6, 10 and 13 , containing some elements $x_{1}, x_{2}, y_{1}$ and $y_{2}$ such that

$$
x_{1} \cup y_{1}=e \quad \text { and } \quad x_{2} \cup y_{2}=e
$$


hold. Then there exist elements $f_{1}$ and $f_{2}$ of $G$ such that $f_{1} x_{1}=f_{2} x_{2}=g$, and that the union $\left(x_{1}: y_{1}\right) \cup\left(x_{2}: y_{2}\right)$ of the arbitrary two right residucals $\left(x_{i}: y_{i}\right)(i=1,2)$ equals to the right residual $\left(g: f_{1} y_{1} \frown f_{2} y_{2}\right)$, i.e.

$$
\left(x_{1}: y_{1}\right) \cup\left(x_{2}: y_{2}\right)=\left(g: f_{1} y_{1} \frown f_{2} y_{2}\right) .
$$

Proof. The negative ordering $y_{1} y_{2} \cup y_{2} y_{1} \leqq y_{1} \frown y_{2}$ for arbitrary $y_{1}, y_{2} \in G$ implies by Axiom 4 evidently $\overline{y_{1}}=\left(y_{1} \frown y_{2}\right): y_{2} \geqq y_{1}$ and $y_{2}=\left(y_{1} \cap y_{2}\right): y_{1} \geqq y_{2}$. Furthermore, by Axiom 10 we have $\bar{y}_{1} \cup y_{2}=e$. Now, Axiom 1 yields $u=e u=\overline{y_{1}} u \cup \overline{y_{2}} u$ for an arbitrary element $u \in G$, whence Axiom 6 gives at once

$$
u:\left(y_{1} \frown y_{2}\right)=\left(u: y_{2}\right) \cup\left(u: y_{1}\right),
$$

substituting into Axiom 6 the elements $x=y_{1} \frown y_{2}, y=y_{i}(i=1,2)$, $z=u$. On the other hand, by Axiom 13 there exist elements $f_{1}$ and $f$ of $G$ for the given $x_{1} \in G$ and $x_{2} \in G$, such that

$$
f_{1} x_{1}=f_{2} x_{2}=g \neq 0
$$

holds. Therefore, for $r_{i}=\left(x_{i}: y_{i}\right)(i=1,2)$, we have

$$
r_{1} \smile r_{2}=\left(f_{1} x_{1}: f_{1} y_{1}\right) \cup\left(f_{2} x_{2}: f_{2} y_{2}\right),
$$

whence the equation (6) yields immediately the desired relation (5), that is:

$$
r_{1} \cup r_{2}=r \text { with } r=\left(g: f_{1} y_{1} \frown f_{2} y_{2}\right) \text { (see (7)). }
$$

This completes the proof of Theorem 15, indeed.

Remarks 16. The requirements (1), Axiom 1, therefore also the negative ordering, furthermore Axiom 4 are satisfied for the 1. o. groupoid $G$ of all twosided ideals of a (not necessarily associative) ring with unity element. The nilpotent rings $A$, for which $A^{n}=0$ for some $n$ holds, show that a ring without unity element has such a l. o. groupoid $G$ in which $e^{2} \neq e$ for its maximum element $e$ holds, therefore Axiom 1 is not satisfied, being now $e=A$. Furthermore, for the l. o. groupoid of all twosided ideals of nilpotent rings $A$, also Axiom 6 fails generally to be valid. On the other hand, the ringtheoretical direct sum $e=x \smile y \smile z$ of three division rings $x, y$ and $z$ shows always $u v=v u=u \frown v$ for $u=x, y$ or $\approx$ and $v=x, y$ or $z$, being the ring $e$ strongly regular (cf. S. LAJos-F. Szász [6]), but generally $u \smile v \neq e$ holds, consequently also Axiom 10 is generally not satisfied for 1 . o. groupoids of twosided ideals of rings. Finally, it can be observed that Axioms 6 and 13 are satisfied by the l. o. semigroup of all twosided ideals of an associative, commutative principal ideal inte-
grity domain.

In what follows, we discuss some examples, which can be considered also as some corollaries for Theorem 15. 
that $f_{1} x_{1}=f_{2} x_{2}=g$, wry two right residuals $\left.\neg f_{2} y_{2}\right)$, i.e.

$y_{1} \cap y_{2}$ for arbitrary $\left(y_{1} \cap y_{2}\right): y_{2} \geqq y_{1}$ and we have $\overline{y_{1}} \cup y_{2}=e$. an arbitrary element

$y_{2}, y=y_{i}(i=1,2)$, ist elements $f_{1}$ and $f_{2}$

re

ad relation (5), that is : I (see (7)).

l.

ffore also the negative 1. o. groupoid $G$ of all $\mathrm{g}$ with unity element. $n$ holds, show that a sid $G$ in which $e^{2} \neq e$ is not satisfied, being all twosided ideals of e valid. On the other $\therefore$ of three division $v$ for $u=x, y$ or $z$ (ef. S. Lajos-F. Szász Axiom 10 is generally rings. Finally, it can $y$ the 1. o. semigroup ' principal ideal intech can be considered
E 1. Let $A$ be a not necessarily associative ring with Axioms 6, 10 and 13 in the 1. o. groupoid of all twosided ideals of $A$, such that $J A=A J=J$ for any twosided ideal $J$ of $A$ holds. If the ideals $B_{1}, B_{2}, C_{1}$ and $C_{2}$ of $A$ satisfy $B_{1}+C_{1}=B_{2}+C_{2}=A$, then one has also

$$
\left(B_{1}: C_{1}\right)+\left(B_{2}: C_{2}\right)=\left(F_{1} B_{1}: F_{1} C_{1} \frown F_{2} C_{2}\right),
$$

where $F_{1} B_{1}=F_{2} B_{2} \neq 0$ for some ideals $F_{1}$ and $F_{2}$ of $A$. Evidently this (9) is a particular case of (5).

E 2. Let $A$ be an associative and commutative principal ideal ring with unity element and without divisors of zero. Then $J A=A J=J$ holds for all ideals. Furthermore $B_{1}+C_{1}=B_{2}+C_{2}=A$ for the twosided ideals $B_{1}, B_{2}, C_{1}$ and $C_{2}$ of $A$ imply by Theorem 15 that

$$
\left(\left(b_{1}\right):\left(c_{1}\right)\right) \cup\left(\left(b_{2}\right):\left(c_{2}\right)\right)=\left(\left(f_{1} b_{1}\right):\left(f_{1} c_{1}\right) \frown\left(f_{2} c_{2}\right)\right)
$$

for some elements $f_{1}$ and $f_{2}$ of $A$, being any ideal $D=(d)$ principal in $A$. Let $(b, c)$ denote the greatest common divisor of $b$ and $c$, furthermore $[b, c]$ the least common multiple of $b$ and $c$. On the other hand, let $F_{0}$ denote the quotient field of the ring $A$ and $\left\{\ldots, b_{\alpha}, \ldots\right\}$ the $A$-submodule generated by the elements ..., $b_{\alpha}, \ldots$ of the $A$-module $F_{0}$. in $A$

Then, for $b_{1}, b_{2}, c_{1}, c_{2} \in A$, the equations $\left(b_{1}, c_{1}\right)=\left(b_{2}, c_{2}\right)=A$ imply

$$
\left(b_{1} c_{2}, b_{2} c_{1}\right)=\left(b_{1}, b_{2}\right) \cdot\left(c_{1}, c_{2}\right),
$$

furthermore in $F_{0}$ also

$$
\left\{\begin{array}{ll}
b_{1} & b_{22} \\
c_{1} & c_{22}
\end{array}\right\}=\left\{\begin{array}{l}
\left(b_{1}, b_{2}\right) \\
{\left[c_{1}, c_{2}\right]}
\end{array}\right\}
$$

Remark 16. A consequence of (12) is that any finitely generated torsion-free commutative group of rank one is cyclic. This follows, on the other hand, also from the fundamental theorem for finitely generated commutative groups (ef. A. G. Kurosh [5]).

E 3. Let $T$ be a groupoid having zero such that the 1. o. groupoid $G$ of all twosided ideals of $\Gamma$ satisfies the Axioms 1, 4, 6, 10 and 13. Let us assume that for twosided ideals $B_{1}, B_{2}, C_{1}$ and $C_{2}$ of $T$ the conditions

$$
B_{1} \cup C_{1}=B_{2} \cup C_{2}=I
$$

hold, where the lattice union $\smile$ coincides naturally with the set theoretical union. Then our Theorem 15 yields:

$$
\left(B_{1}: C_{1}\right) \cup\left(B_{2}: C_{2}\right)=\left(F_{1} B_{1}:\left(F_{1} C_{1} \frown F_{2} C_{2}\right)\right)
$$

for some twosided ideals $F_{1}$ and $F_{2}$ of the groupoid $I$.

E 4. Let now $T$ be, in particular, an arbitrary group, furthermore let $G$ be the lattice of all its normal subgroups, such that $G$ by the building of 
mutual commutator subgroups $\left[N_{1}, N_{2}\right]$ generated in $I$ by all commutators $n_{1}^{-1} n_{2}^{-1} n_{1} n_{2}$ with $n_{1} \in N_{1}$ and $n_{2} \in N_{2}$ becomes also a 1 . o. groupoid. Let us assume that Axioms 1, 4, 6, 10 and 13 for $G$ are satisfied. Then

$$
B_{1} \cup C_{1}=B_{2} \cup C_{2}=I
$$

imply by our 'Theorem 15 that

$$
\left(B_{1}: C_{1}\right) \cup\left(B_{2}: C_{2}\right)=\left(\left[F_{1}, B_{1}\right]:\left(\left[F_{1}, C_{1}\right] \frown\left[F_{2}, C_{2,}\right]\right)\right)
$$

with some normal subgroups $F_{1}, F_{2} \in G$, and for the normal subgroups $B_{1}, B_{2}, C_{1}$ and $C_{2}$ of $T$ holds. Here one has, by definition, for normal subgroups $B$ and $C$ of $\Gamma$

$$
B: C=\left\{x ; x \in T, x^{-1} c^{-1} x c \in B \quad \text { for any } \quad c \in C\right\},
$$

furthermore $B \cup C$ is the normal subgroup of $I$, generated by all group products $b c$ with $b \in B, c \in C$. On the other hand $B \cap C$ is the set theoretical intersection.

$\mathbf{E}$ 5. Let $\Gamma$ be a group, such that with the notions discussed at $\mathbf{E} 4$, $[T, N]=N$ for any normal subgroup $N$ of $I$ holds. Furthermore, let us assume that the group $T$ is a so-called „principal normal subgroup integrity domain" in the sense that for any normal subgroup $N$ of $T$ there exists an element $\gamma \in \Gamma$ such that $N=[\gamma, \Gamma]$ holds, furthermore

$$
\left[N_{1}, N_{2}\right]=\{1\} \quad \text { implies } N_{1}=\{1\} \quad \text { or } \quad N_{2}=\{1\} \text {, }
$$

where $\{1\}$ is the unity subgroup of $I$. Then, for the 1 . o. groupoid $G$, with operations of $\mathbf{E} 4$, of all normal subgroups of $I$, all Axioms 1, 4, 6, 10 and 13 are satisfied. Therefore $\mathbf{E} 5$ is a particular case of $\mathbf{E} 4$, consequently our Theorem 15 can be applied to $\mathbf{E} \mathbf{5}$.

Obviously, any simple noncommutative group belongs to the class $\boldsymbol{C}_{0}$, discussed at $\mathbf{E} \mathbf{5}$.

Furthermore, if a group of this class $\boldsymbol{C}_{0}$ is meta-abelian, that is the condition

$$
[I,[I, I]]=\{1\}
$$

holds, then the 1. o. groupoid $G$ for $T$ is, by A. G. Kunosm [5], a semigroup, because then one has $[x,[y, z]]=[[x, y], z]=1$ for every element

$$
x, y, z \in T \text {. }
$$

Remark 17. A very particular form of Theorem 1.5, when we have discussed some (principal) ideals of the ring $J$ of all rational integers, has occurred ten years ago on page 130 of author's paper [14].

Finally, we point out some open problems:

$P$ 1: Can be given a generalization of Theorem 15 for categories $C$, satisfying some axioms (e.g. the normal subobjects of any object of $C$ form a set, which is a compactly generated complete lattice etc., and yet similar axioms to Axioms 1, $[15]))$ ?

$P 2$ : What is a ne groupoid $G$ that any : again a right residua:

P 3: Let us inves1 integrity domain gror the same time also [I (E. g. is the class $\boldsymbol{C}_{0}$ C ducts, or to building [15]). Or, what is the 1 ,

[1] B. A. Апдрунакценп мax с частпымі. ІІзг

[2] G. Bгккноғғ, Lattice

[3] L. Fucins, Partially O

[4] N. Jacobsox, Structu

[5] A. I. Kypom, Teopur.

[6] S. Lajos-F. Szász, So $31,223-228(1970)$.

[7] L. Lesieck, Sur les t1 Sci. Paris 232, 290-2

[8] O. Strinfeld, Verbar Acta Sci. Math. 2o, 1:

[9] - , On residuals in pa (1965).

[10] - , Utber Gruppoid-Vc

[11] II. В. Стелтециниі. $\mathrm{F}$ 211-235 (1960).

[12] E. I. IIIульеïфер, P нпем, У

[13] F. Szász, Verbandsth nichtassoziatischen $\mathrm{Ri}$

[14] -, Die Ringe, deren Acta Math. Acad. Sci.

[15] F. SzÁsz-R. WIEGAND' Acad. Sci. Hungar. 20

[16] G. SzÁsz, Introductior

[17] M. WARD, Residuatio math. J. 3, 627-636 (

[18] M. WARD-R. P. DILWC 354 (1939).

Magyar Tudomanyos Matematikai Kutato I Budapest V, Reáltuno 
joids

by all commutators o. groupoid. Let us ied. Then

$\left.\left.>\left[F_{2}, C_{2}\right]\right)\right)$

normal subgroups on, for normal sub-

$c \in C\}$,

rated by all group $C$ is the set theore-

is discussed at $\mathbf{E} 4$, Eurthermore, let us 'mal subgroup interoup $N$ of $I$ there furthermore

$$
N_{2}=\{1\},
$$

o. groupoid $G$, with Axioms 1, 4, 6, 10 if $\mathbf{E} 4$, consequently ngs to the class $C_{0}$, Ibelian, that is the

SH [5], a semigroup, every element

15, when we have ttional integers, has : [14].

5 for categories $C$, ny object of $C$ form ste., and yet similar
Szász, On right residuals in lattice ordered groupoids

axioms to Axioms 1, 4, 6, 10 and 13 hold (cf. c. g. F. Szász-R. WHEGAxdT $[15])) ?$

P 2: What is a necessary and sufficient condition for a 1. o. residuated groupoid $G$ that any finite (or any infinite) union of right residuals let bo again a right residual of $G$ ?

$P$ 3: Let us investigate more in detail the ,principal normal subgroup integrity domain groups" $T$, discussed at our example $\mathbf{E} \mathbf{5}$, for which at the same time also $[I, N]=N$ with any normal subgroup $N$ of $I$ holds! (E. g. is the class $\boldsymbol{C}_{0}$ of these groups closed, with respect to subdirect products, or: to building of ,trans-free images"? (cf. F. Szísz-R. WIEGANDT [15]). Or, what is the lower radical class determined by this class of groups?)

\section{References}

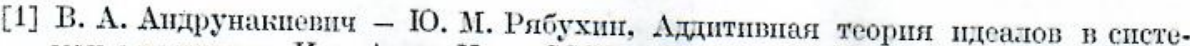
мах с частыми. ІІзв. Акад. Наук СССР, сер. математ. 31, 1057-1090 (1967).

[2] G. Brкnofr, Lattice Theory. Providence 1956.

[3] L. Fuchs, Partially Ordered Algebraic Systems. Oxford-London-New York-Paris 1963.

[4] N. Jacossox, Structure of Rings, 2nd edition. Providence 1964.

[5] А. T. Куроп, Теopпr групा, Москва, 1967.

[6] S. LaJos-F. Szász, Some characterizations of twosided regular rings, Acta Sci. Math. $31,223-228(1970)$.

[7] L. Lestevr, Sur les treillis multiplicatifs complets à condition minimale, C.R. Acad. Sci. Paris 232, 290-292 (1951).

[8] O. Stmixferd, Verbandstheoretische Betrachtung gewisser ideal-theoretischer Fragen, Acta Sci. Math. 22, 136-149 (1961).

[9] -, On residuals in partially ordered semigroups, Publ. Math. Debrecen 12, 107-116 (1965).

[10] -, U゙ber Gruppoid-Verbände, I, Acta Sci. Mrath. 31, 203-218 (1970).

[11] II. В. Стеллецинй. Нилпотенные структуры, Труды Москов. Математ. Общ. 9, 211-235 (1960).

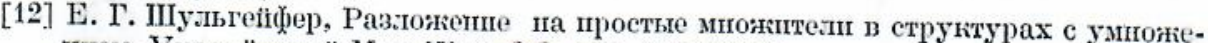

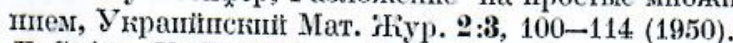

[13] F. Szász, Verbandstheoretische Bemerkungen zum Fuchsschen Zeroidradikal der nichtassoziatischen Ringe, Archiv der Math. 12, 282-289 (1961).

[14] - Die Ringe, deren endlich erzeugbare echte Unterringe Hauptrechtsideale sind, Acta Math. Acad. Sci. Hungar. 13, 115-132 (1962).

[15] F. Szász-R. Wregaxd, On the dualization of subdirect embeddings, Acta Math. Acad. Sci. Hungar. 20, 289-302 (1969).

[16] G. Szász, Introduction to Lattice Theory. Budapest 1963.

[17] M. WARD, Residuation in structures over which a multiplication is defined, Duke math. J. 3, 627-636 (1937).

[18] MI. Ward-R. P. Dilwontr, Residuated lattices, Trans. Amer. Math. Soc. 45, 335 bis 354 (1939).

\author{
Magyar Tulomanyos Akademia \\ Matematikai Kutato Intesete \\ Budapest V, Reciltanoda u. 13-1.5
}

